\title{
Charge Retention Improvement of Nonvolatile Radiation Sensor Using Metal-Oxide-Nitride-Oxide-Silicon with Si-Rich Nitride and Oxy-Nitride as Stack Charge-Trapping Layer
}

\author{
Wen-Ching Hsieh, ${ }^{*}$ Hao-Tien Daniel Lee, ${ }^{1}$ Fuh-Cheng Jong, ${ }^{2}$ and Shich-Chuan $\mathrm{Wu}^{3}$ \\ Department of Opto-Electronic System Engineering, Minghsin University of Science and Technology, \\ Xinxing Road 1, Xinfeng 30401, Taiwan \\ ${ }^{1}$ Treasure Giant Technology Inc. 3F-1, 42 Lyushuei Road, Hsinchu 30068, Taiwan \\ ${ }^{2}$ Electronic Engineering Department, Southern Taiwan University of Science and Technology, \\ 1, Nan-Tai Street, Yungkang District, Tainan 710, Taiwan \\ ${ }^{3}$ National Nano Device Laboratories, No. 26, Prosperity Road 1, Hsinchu Science Park, Hsinchu 30078, Taiwan
}

(Received December 28, 2015; accepted June 10, 2016)

Keywords: high k, sensor, SONOS, MOS, radiation

Metal-oxide-nitride-oxide-silicon devices with Si-rich nitride and oxy-nitride as a bi-layer stack charge-trapping layer (hereafter STOB-MONOS) could be candidates for nonvolatile total ionizing dose (TID) radiation sensors. In the case of STOB-MONOS nonvolatile TID radiation sensors, gamma radiation induces a significant decrease in the threshold voltage $V_{\mathrm{T}}$, which is nearly 2 times larger than that of a standard MONOS device. The change in $V_{\mathrm{T}}$ for STOB-MONOS after gamma irradiation also has a strong correlation to TID up to $10 \mathrm{Mrad}$ gamma irradiation. The reliability characteristics of $V_{\mathrm{T}}$ retention time before and after gamma irradiation for STOBMONOS devices can be markedly improved and is nearly $12 \%$ better than that of a standard MONOS device. The STOB-MONOS device in this study has demonstrated the possibility of improved the feasibility of non-volatile high TID radiation sensing.

\section{Introduction}

The measurement of the total ionizing dose (TID) is a major concern in various radiation applications, such as space radiation monitoring and advanced X-ray lithography semiconductor manufacturing processes. Semiconductor dosimeters offer many advantages over other commonly used dosimeters, such as ionization chambers and thermoluminescent dosimeters (TLDs). The sensing areas of semiconductor dosimeters are very small, and their sensitivity can be very high in a small constrained space. A silicon-silicon dioxide-silicon nitride-silicon dioxide-silicon (SONOS) device has been shown to be suitable for nonvolatile, high TID radiation sensor applications. ${ }^{(1,2)}$ The ionizing radiation induces ionized electron-hole pairs in the SONOS device, and positive charges are trapped in the charge-trapping layer of the nonvolatile SONOS device. The build-up of positive charge changes the threshold voltage $V_{\mathrm{T}}$, and the $V_{\mathrm{T}}$ shift depends on the absorbed TID of the ionizing radiation. The SONOS device can store accumulated TID information

"Corresponding author: e-mail: wchsieh@must.edu.tw 
permanently. ${ }^{(1-8)}$ However, the charge-retention reliability improvement of a SONOS device after high TID gamma irradiation has not been well studied; it is discussed in this study. A metaloxide-nitride-oxide-silicon device with Si rich nitride at the top and oxy-nitride at the bottom of the charge-trapping layer (hereafter STOB-MONOS) was proposed in this study. The radiationinduced charging effects and charge-retention reliability of the STOB-MONOS devices were significantly improved. The electrical performance of the STOB-MONOS devices with various $\mathrm{Si}-$ $\mathrm{O}-\mathrm{N}$ composition ratios after gamma irradiation, including radiation-induced charge density, gate leakage current and charge retention reliability, were the main subjects of this study.

\section{Experimental Details}

STOB-MONOS devices prepared by adjusting the $\mathrm{Si}-\mathrm{O}-\mathrm{N}$ composition ratio during nitride deposition for this study are listed in Table 1. MONOS structures were fabricated on p-type resistivity $15-25 \Omega-\mathrm{cm} \mathrm{Si}<100>$ substrate. We used thermal $\mathrm{SiO}_{2}$ for the tunneling oxide, chemical vapor deposition (CVD) nitride $\mathrm{Si}_{3} \mathrm{~N}_{4}$ for the trapping layer, and CVD TEOS $\mathrm{SiO}_{2}$ for the blocking oxide of the $\mathrm{SiO}_{2}-\mathrm{Si}_{3} \mathrm{~N}_{4}-\mathrm{SiO}_{2}(\mathrm{ONO})$ gate dielectric. The tunneling silicon oxide $\left(\mathrm{SiO}_{2}\right)$ was formed on the wafers by an advanced clustered vertical furnace. After the tunneling oxide was formed, silicon nitride (hereafter, nitride) $\left(\mathrm{Si}_{3} \mathrm{~N}_{4}\right)$ was deposited as the charge-trapping layer by low-pressure chemical vapor deposition (LPCVD) on the MONOS device. Four types of MONOS devices prepared by adjusting the gas flow-rate ratio of $\mathrm{SiH}_{2} \mathrm{Cl}_{2}-\mathrm{NH}_{3}$ and $\mathrm{SiH}_{2} \mathrm{Cl}_{2}-\mathrm{N}_{2} \mathrm{O}$ during charge-trapping nitride film deposition were compared, as shown in Table 1: (1) "STD" type MONOS (hereafter STD-MONOS) with standard nitride $\left(\mathrm{SiH}_{2} \mathrm{Cl}_{2}: \mathrm{NH}_{3}=0.25: 1\right)$ as the chargetrapping layer; (2) "S" type MONOS (S-MONOS) with Si-rich nitride $\left(\mathrm{SiH}_{2} \mathrm{Cl}_{2}: \mathrm{NH}_{3}=2: 1\right)$ as the charge-trapping layer; (3) "O" type MONOS (O-MONOS) with oxy-nitride $\left(\mathrm{SiH}_{2} \mathrm{Cl}_{2}: \mathrm{NH}_{3}=2: 1\right.$ and $\mathrm{SiH}_{2} \mathrm{Cl}_{2}: \mathrm{N}_{2} \mathrm{O}=0.15: 1$ ) as the charge-trapping layer; and (4) "STOB" type MONOS (STOB-MONOS) with a bi-layer stacked trapping layer of silicon-rich nitride $\left(\mathrm{SiH}_{2} \mathrm{Cl}_{2}: \mathrm{NH}_{3}=2: 1\right)$ on the top and oxy-nitride $\left(\mathrm{SiH}_{2} \mathrm{Cl}_{2}: \mathrm{NH}_{3}=2: 1\right.$ and $\left.\mathrm{SiH}_{2} \mathrm{Cl}_{2}: \mathrm{N}_{2} \mathrm{O}=0.15: 1\right)$ on the bottom. The $\mathrm{ONO}$ gate stack consisted of a 100-200 $\AA$ silicon nitride and 50-150 $\AA$ bottom and top silicon oxides. The TiN metal gate $(200-400 \mathrm{~nm})$ was formed by DC sputtering for the control gate. After gate patterning, the source and drain were formed by implantation of arsenic atoms, which were activated at $900{ }^{\circ} \mathrm{C}$ for $30 \mathrm{~s}$. Figure 1(a) shows the cross-section view of the MONOS devices. For comparison, all the devices listed in Table 1 had the same thickness of tunneling oxide, trapping nitride, and blocking oxide layer.

For gamma TID data writing, ${ }^{60} \mathrm{Co}$ gamma radiation was impinged on these four type MONOS devices at a negative gate bias stress (NVS) $\left(V_{\mathrm{G}}=-5 \mathrm{~V}\right)$. For the gamma TID data read, $V_{\mathrm{T}}$ was measured at room temperature using a HP4156A parameter analyzer. The experimental results of

Table 1

Four types of MONOS devices with various charge-trapping layer.

\begin{tabular}{lcc}
\hline Split & Charge-trapping layer structure & Trapping layer thickness \\
\hline STD & Standard nitride (single layer) & $20 \mathrm{~nm}$ \\
S & Si-rich nitride (single layer) & $20 \mathrm{~nm}$ \\
O & Oxy-nitride (single layer) & $20 \mathrm{~nm}$ \\
STOB & Si-rich nitride on top and oxy-nitride on bottom (bilayer) & $10 \mathrm{~nm}$ top and $10 \mathrm{~nm}$ bottom \\
\hline
\end{tabular}




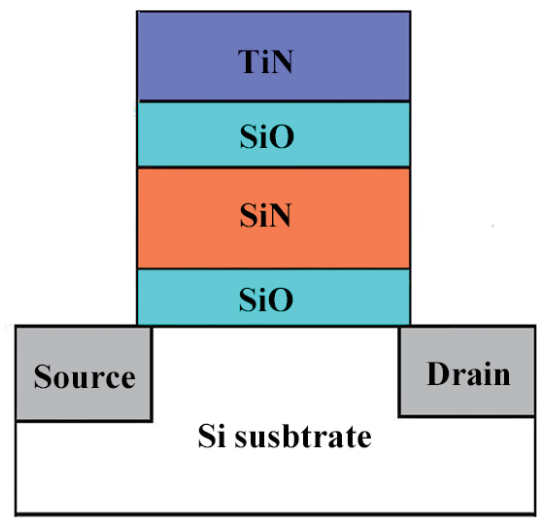

(a)

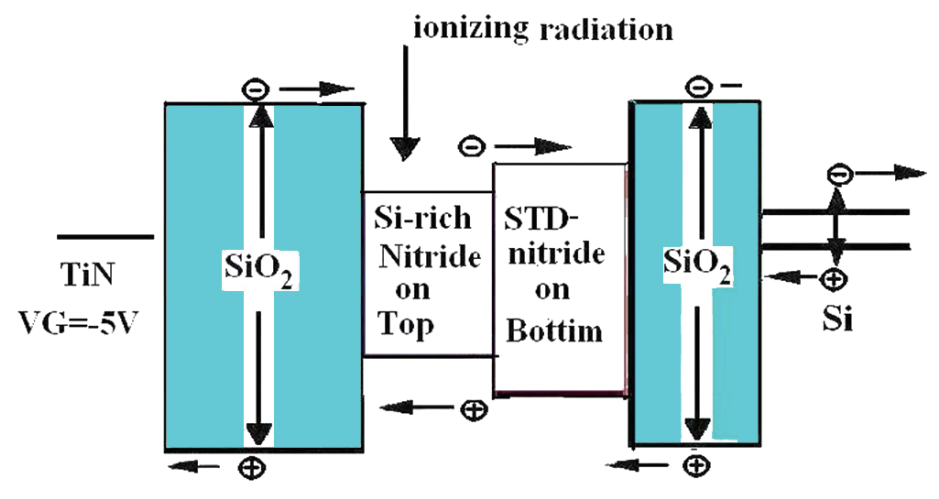

(b)

Fig. 1. (Color online) (a) Cross-sectional view of an STD-MONOS device. (b) Charge generation and trapping in the STOB-MONOS after gamma irradiation.

gate capacitance applied at various gate voltages $\left(C_{\mathrm{G}}-V_{\mathrm{G}}\right)$ were obtained by a computer-controlled HP4284 parameter analyzer, and the $C_{\mathrm{G}}-V_{\mathrm{G}}$ curves were measured by sweeping $V_{\mathrm{G}}$ together with zero source-and-drain bias conditions at room temperature. Figure 1(b) shows the charge generation and trapping states of the gate dielectric in the STOB-MONOS device after gamma irradiation.

\section{Results and Discussion}

\subsection{Radiation-induced $V_{\mathrm{T}}$ shift in STOB-MONOS after gamma irradiation}

Figure 2(a) shows a $C_{\mathrm{G}}-V_{\mathrm{G}}$ curve for a typical MONOS device with NVS $\left(V_{\mathrm{G}}=-5 \mathrm{~V}\right)$ processed with gamma irradiation up to $10 \mathrm{Mrad}$ TID. As illustrated in Fig. 2(a), the $C_{\mathrm{G}}-V_{\mathrm{G}}$ curve of STDMONOS shifted to the left after 10 Mrad TID of gamma irradiation. This implies that gamma irradiation induces a decrease of $V_{\mathrm{T}}$ for STD-MONOS. The amount of the decrease in $V_{\mathrm{T}}$ is about 2.25 volts. This negative $V_{\mathrm{T}}$ shift result is in agreement with previous studies. ${ }^{(1-8)}$ The change is due to an increase in net positive trapped charges in the ONO gate dielectric layer after gamma irradiation. These radiation-induced shifts in the irradiated device result from a combination of two effects: the first, from the loss of stored negative charge in the ONO trapping layer; the second from a build-up of positive charge resulting from asymmetric trapping of electrons and holes in the ONO trapping layer. ${ }^{(1-8)}$ The TID radiation induced-charging effect of the device was determined by the survival yield of an electron-hole pair, which was a portion of free holes and electrons that survived after the recombination process immediately following the excitation due to ionizing radiation. ${ }^{(9)}$

As shown in Fig. 2(b), the $C_{\mathrm{G}}-V_{\mathrm{G}}$ curve of STOB-MONOS shifted far to the left after 10 Mrad gamma irradiation. The amount of decrease in $V_{\mathrm{T}}$ was up to about $4.5 \mathrm{~V}$. As shown in the experiment data, the change of $V_{\mathrm{T}}$ for STOB-MONOS is more significant than that for STDMONOS after $10 \mathrm{Mrad}$ of gamma irradiation. The amount of stored negative charge lost and net positive charges build-up due to gamma irradiation in the STOB-MONOS was greater than that in the STD-MONOS after gamma irradiation. 


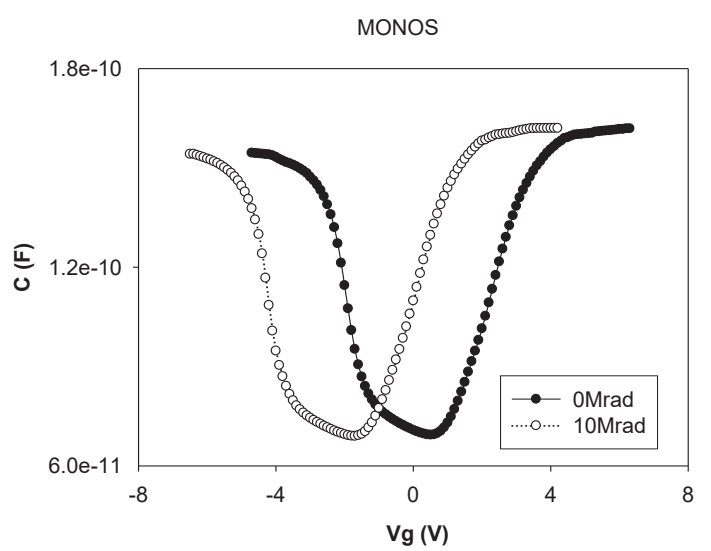

(a)

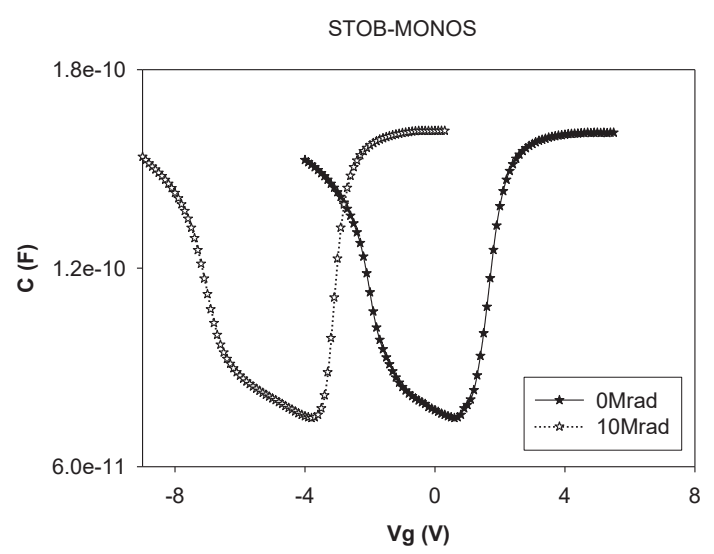

(b)

Fig. 2. (a) The $C_{\mathrm{G}}-V_{\mathrm{G}}$ curve for an STD-MONOS device after 10 Mrad gamma irradiation. (b) The $C_{\mathrm{G}}-V_{\mathrm{G}}$ curve for an STOB-MONOS device after 10 Mrad gamma irradiation.

\section{2 $\quad V_{\mathrm{T}}$ decay vs TID in STOB-MONOS after gamma irradiation}

In both Figs. 3(a) and 3(b), the decrease in $V_{\mathrm{T}}$ for STD-MONOS and STOB-MONOS devices under $V_{\mathrm{G}}=-5 \mathrm{~V}$ are plotted against the TID of gamma irradiation. The decay of $V_{\mathrm{T}}$ for STDMONOS and STOB-MONOS devices increased as a function of gamma TID is indicated in Figs. 3(a) and 3(b). The decrease in $V_{\mathrm{T}}$ of STD-MONOS shown in Fig. 3(a) can be correlated to the increase in gamma TID and the increase in positive trapped charges in the insulator as well. The decay of $V_{\mathrm{T}}$ increased more sharply after gamma irradiation levels up to $100 \mathrm{krad}$ TID. These experimental results in this study are in agreement with previous studies..$^{(1-8)}$

The dependence of the $V_{\mathrm{T}}$ shift on gamma TID for STOB-MONOS was more obvious than that for STD-MONOS after gamma irradiation, as shown in Fig. 3(b). The increase in radiation-induced $V_{\mathrm{T}}$ shift in STOB-MONOS can be explained by the increase of radiation-induced net positive charges in STOB-MONOS compared to that in STD-MONOS.

The comparison of the radiation-induced $V_{\mathrm{T}}$ decrease on gamma TID for four types of MONOS devices is shown in Fig. 3(c). The radiation-induced charge density can be calculated from delta $V_{\mathrm{T}}$ using the Terman method.(10) The dependence of the radiation-induced $V_{\mathrm{T}}$ decrease on gamma TID for S-MONOS and O-MONOS was more obvious than that for STD-MONOS after gamma irradiation, as shown in Fig. 3(c). The amount of radiation-induced positive-charge build-up due to gamma irradiation in the silicon-rich nitride or oxy-nitride is greater than that in the standard nitride. The dependence of radiation-induced $V_{\mathrm{T}}$ decrease on gamma TID for STOB-MONOS was more obvious than that for S-MONOS and O-MONOS after gamma irradiation, as shown in Fig. 3(c). The amount of radiation-induced positive-charge build-up due to gamma irradiation in the bilayer stack trapping layer (silicon-rich nitride on top and oxy-nitride on bottom) is greater than that in the entire single-layer Si-rich nitride or single-layer oxy-nitride. Note that the radiationinduced $V_{\mathrm{T}}$ decrease of the STOB-MONOS device was 2 times larger than that of the STDMONOS device. The increase in radiation-induced $V_{\mathrm{T}}$ shift in STOB-MONOS can be explained by the increase of radiation-induced net positive charge in STOB-MONOS compared to that in STDMONOS. 


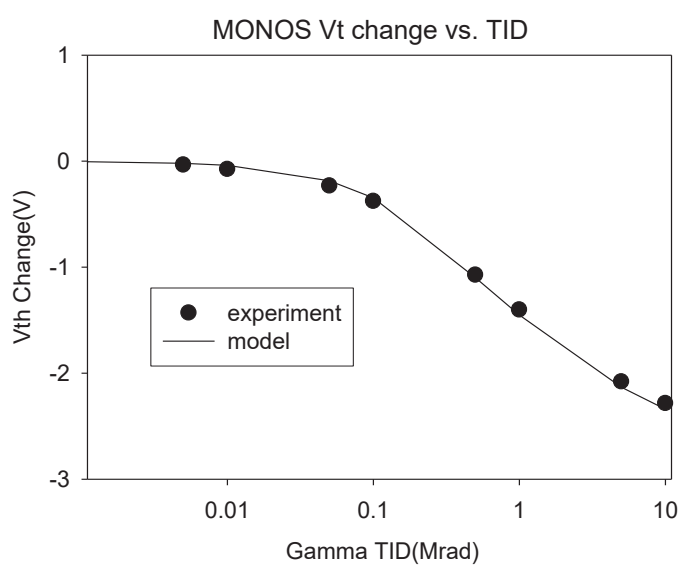

(a)

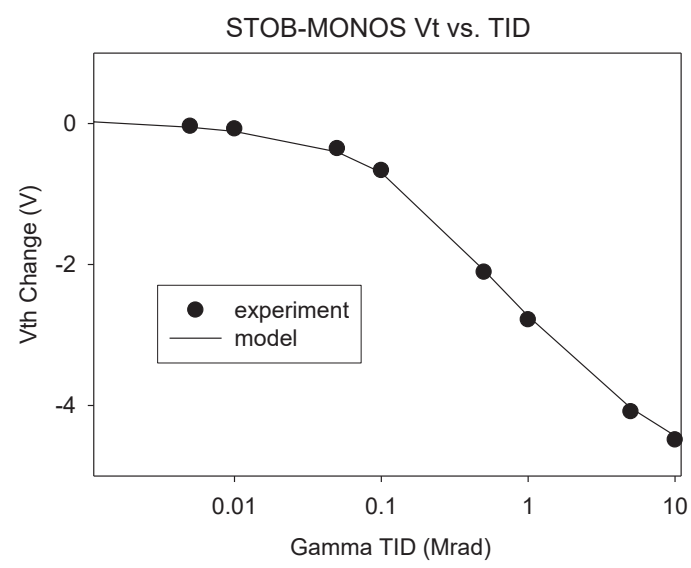

(b)

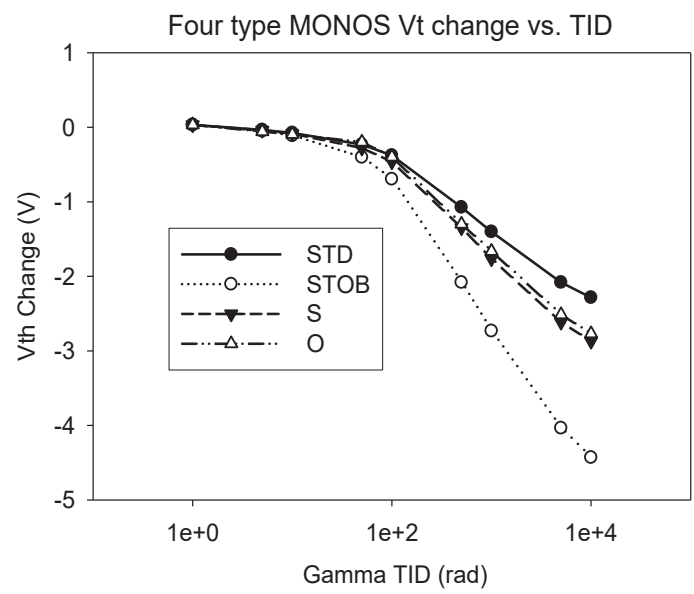

(c)

Fig. 3. (a) The dependence of the $V_{\mathrm{T}}$ decrease on gamma TID for an STD-MONOS device. (b) The dependence of the $V_{\mathrm{T}}$ decrease on gamma TID for an STOB-MONOS device. (c) The dependence of the $V_{\mathrm{T}}$ decrease on gamma TID for four types of MONOS devices.

\subsection{Charge loss vs annealing temperature}

The $V_{\mathrm{T}}$ loss from the pre-irradiated device as a function of annealing temperature was investigated to confirm the effect of rich incorporation of $\mathrm{Si}$ and $\mathrm{O}$ on gate oxide leakage current and charge retention reliability characteristics of MONOS, as shown in Fig. 4. As illustrated in Fig. 4, a much shallower negative-charge trap energy level $E_{\mathrm{TA}}$ is observed for S-MONOS than that for STD-MONOS. This result also agreed with the previous study for the Si-rich nitride chargetrapping layer in a MONOS device. ${ }^{(11,12)}$ Relatively shallow negative-charge trap energy levels $\left(E_{\mathrm{TA}}\right)$ originating from $\mathrm{Si}-\mathrm{H}$ or Si dangling bonds were observed for S-MONOS (compared to STDMONOS). This result also agreed with previous studies for a MONOS device with a Si rich nitride charge-trapping layer. ${ }^{(11,12)}$ Furthermore, a much deeper negative-charge $E_{\mathrm{TA}}$ was observed for O-MONOS with an oxy-nitride trapping layer than for STD-MONOS. This result also agreed with the previous study of a MONOS device with an oxy-nitride charge-trapping layer. ${ }^{(11,12)}$ 


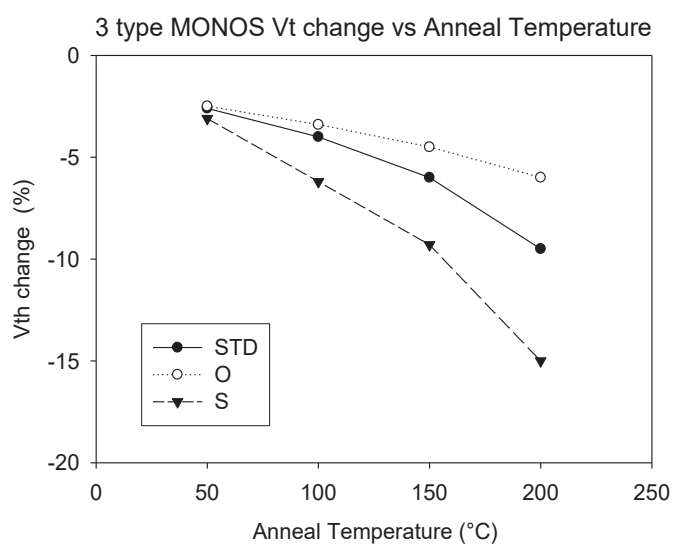

Fig. 4. The $V_{\mathrm{T}}$ decay vs annealing temperature for three types of MONOS device.

\subsection{Measurement of gate leakage current}

As illustrated in Figs. 5(a) and 5(b), the gate oxide leakage current of STOB-MONOS and STDMONOS devices did not increase significantly after $10 \mathrm{Mrad}$ gamma irradiation. The gate oxide leakage current of STOB-MONOS increased more significantly than STD-MONOS after $10 \mathrm{Mrad}$ gamma irradiation.

\subsection{Model for $V_{\mathrm{T}}$ shift under various TID}

The experimental results for STD-MONOS and STOB-MONOS devices [as illustrated in Figs. 3(a) and 3(b)] can be fit well by the HWC model. ${ }^{(1)}$ The HWC model can be used to model the $V_{\mathrm{T}}$ shift of SONOS type devices under various irradiation conditions: ${ }^{(1)}$

$$
V_{\mathrm{T}}(D)=\left[V_{\mathrm{T}}(0)-A\right]\left\{[B] \cdot \exp \left(-t_{\mathrm{n}} D\right)+[1-B] \cdot \exp \left(-t_{\mathrm{p}} D\right)\right\}+A
$$

Equation (1) also can be written as

$$
V_{\mathrm{T}}(D)=A \cdot[1-C(D)]+V_{\mathrm{T}}(0) \cdot C(D)
$$

where

$$
\left.C(D)=[B] \cdot \exp \left(-t_{\mathrm{n}} D\right)+[1-B] \cdot \exp \left(-t_{\mathrm{p}} D\right)\right\}
$$

Delta $V_{\mathrm{T}}(D)$ can be derived from Eqs. (1) and (2) as

$$
\text { Delta } V_{\mathrm{T}}(D)=V_{\mathrm{T}}(D)-V_{\mathrm{T}}(0)=\left[V_{\mathrm{T}}(0)-A\right] \cdot[C(D)-1],
$$

where " $D$ " represents the TID. The terms " $t_{\mathrm{n}}$ " and " $t_{\mathrm{p}}$ " are defined as the sum of emission and capture constant of electrons and holes, respectively. The term " $A$ " is the constant for specific device, and " $B$ " is the combination ratio of electrons and holes. The analysis showed that $t_{\mathrm{n}}=$ 


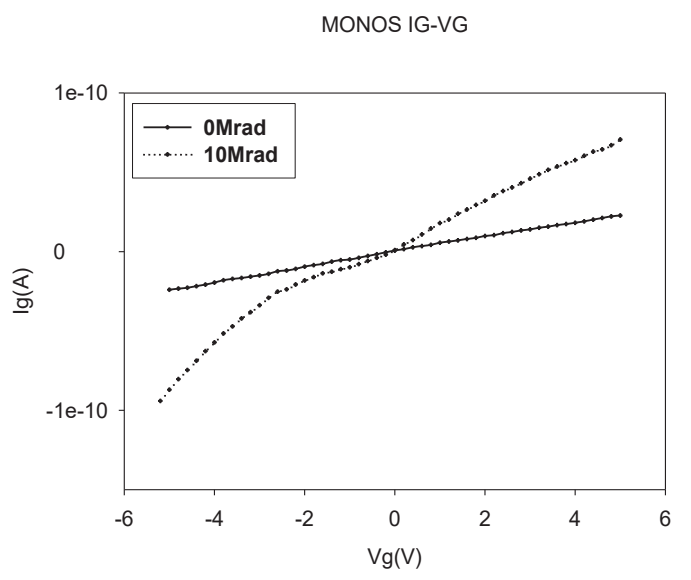

(a)

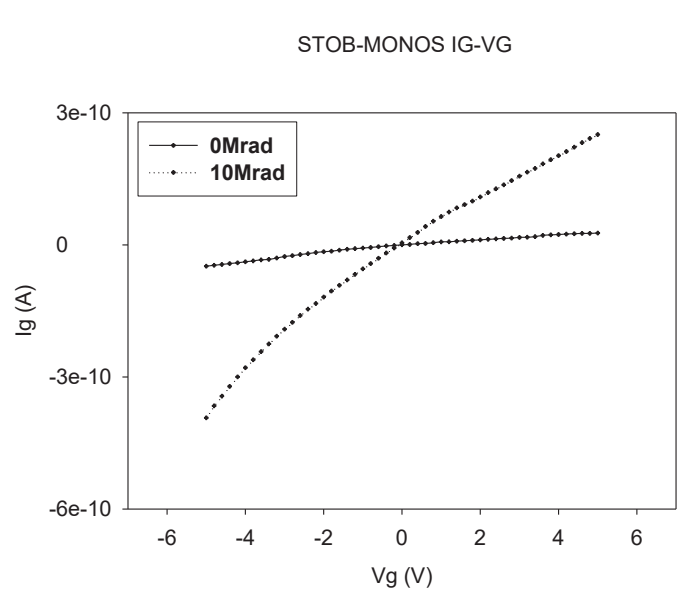

(b)

Fig. 5. (a) The gate-current for an STD-MONOS device after 10 Mrad gamma irradiation. (b) The gate-current for STOB-MONOS device after 10 Mrad gamma irradiation.

$2.5 \mathrm{E}-3 / \mathrm{krad}, t_{\mathrm{p}}=2.5 \mathrm{E}-4 / \mathrm{krad}, B=0.5$, and $A=-1.8$ is the optimum fitting parameter to predict the radiation response of the STD-MONOS device in Fig. 3(a). However $A=-4.1$ is the optimum fitting parameter for radiation response of the STOB-MONOS devices in Fig. 3(b). The increase in " $A " \mid$ for STOB-MONOS devices can be explained because the increase of radiation-induced positive charge in the STOB-MONOS devices is greater than that in the STD-MONOS device after gamma irradiation. Figures 3(a) and 3(b) show graphic views of the results from the HWC model plotted against the experimental data for STD-MONOS and STOB-MONOS devices. This shows that the HWC model can fit the experimental data well.

\section{6 $\quad V_{\mathrm{T}}$ stability vs retention time}

The $V_{\mathrm{T}}$ stability vs time for STOB-MONOS under $V_{\mathrm{G}}=-5 \mathrm{~V}$ before gamma irradiation and after $10 \mathrm{Mrad}$ gamma irradiation is illustrated in Figs. 6(a) and 6(b) respectively. The decrease in $V_{\mathrm{T}}$ with time for the pre-irradiated MONOS device is a result of stored negative charges tunneling out from the nitride trapping layer, and the increase in the $V_{\mathrm{T}}$ with time for the post-irradiated MONOS device is a result of radiation-induced positive charges tunneling out from the nitride trapping layer. However, STOB-MONOS demonstrated better charge-retention reliability characteristics than STDMONOS, both before gamma irradiation and after 10 Mrad gamma irradiation.

Figures 7(a) and 7(b) show the charge retention reliability characteristics of four types of MONOS devices before gamma irradiation and after $10 \mathrm{Mrad}$ gamma irradiation. The loss of stored negative charge from the S-MONOS device before gamma irradiation was worse than that of the STD-MONOS, as shown in Fig. 5(a). Consequently, the rate of charge-loss from the preirradiated device as a function of annealing temperature was investigated to confirm the effect of rich $\mathrm{Si}$ and $\mathrm{O}$ incorporation on charge retention reliability characteristics of MONOS. For preirradiated S-MONOS, a larger negative-charge-loss with retention time was observed due to the relatively shallow negative-charge trap energy level $\left(E_{\mathrm{TA}}\right)$ originating from $\mathrm{Si}-\mathrm{H}$ or $\mathrm{Si}$ dangling bonds, which causes significant thermal excitation of trapped negative charges to the nitride 


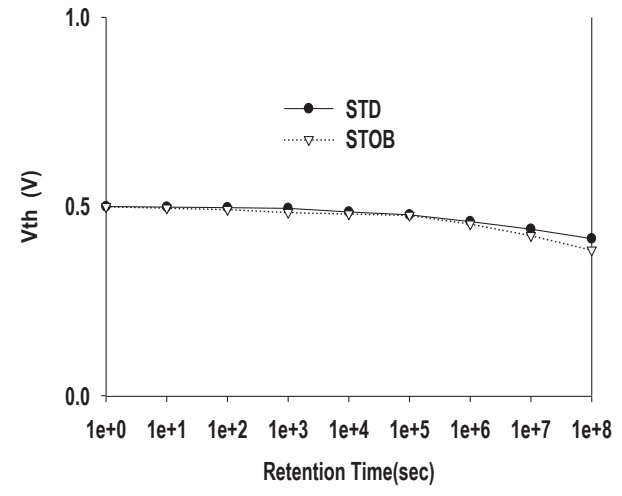

(a)

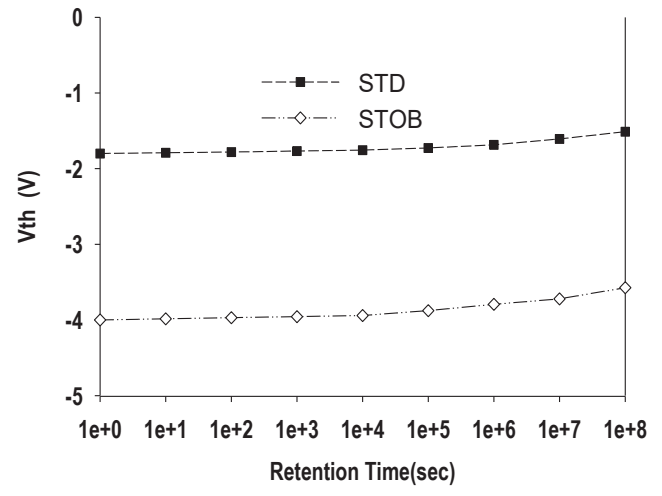

(b)

Fig. 6. The $V_{\mathrm{T}}$ vs retention time for a STOB-MONOS device: (a) before gamma irradiation and (b) after 10 Mrad gamma irradiation.

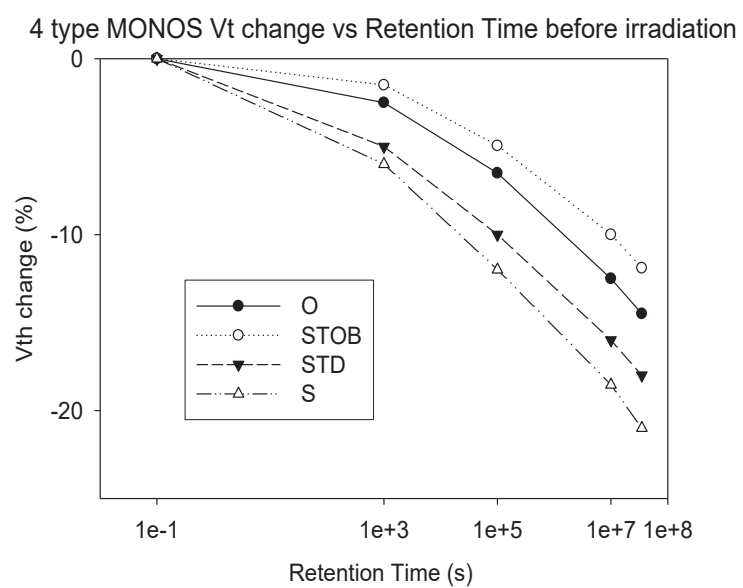

(a)

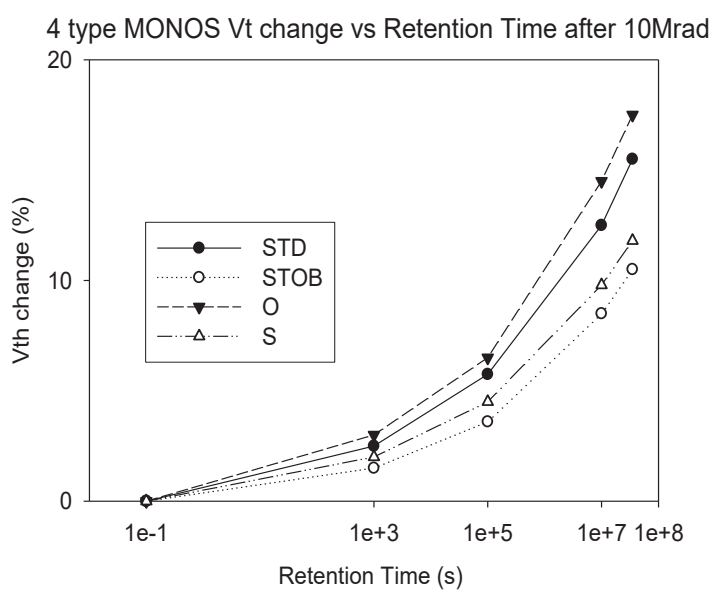

(b)

Fig. 7. The $V_{\mathrm{T}}$ decay vs time for four type MONOS under $V_{\mathrm{G}}=-5 \mathrm{~V}$ after (a) 0 Mrad gamma irradiation and (b) 10 Mrad gamma irradiation.

conduction band. ${ }^{(11,12)}$ The result shows that high silicon composition ratio in the trapping nitride layer led to worse negative charge retention reliability characteristic of pre-irradiated S-MONOS. The figure shows that the trend in charge retention loss with time for S-MONOS after $10 \mathrm{Mrad}$ gamma irradiation is exactly opposite to that before gamma irradiation. The result shows that a high silicon composition ratio and relatively shallow negative charge $E_{\mathrm{TA}}$ in the trapping nitride layer led to worse negative charge retention reliability characteristics for pre-irradiated S-MONOS, and better positive charge retention reliability characteristics for 10 Mrad gamma irradiated S-MONOS (compared to that of STD-MONOS). However, STOB-MONOS demonstrated better charge-retention reliability characteristics than STD-MONOS both before gamma irradiation and after $10 \mathrm{Mrad}$ gamma irradiation. Before gamma irradiation, the probability of stored negative charges tunneling out from the bottom of the trapping nitride to the tunneling oxide was higher (compared to that from the top of trapping nitride to blocking oxide) for the pre-irradiated MONOS device under NVS. After $10 \mathrm{Mrad}$ gamma irradiation, the probability of radiation-induced positive charges tunneling out from the top of the trapping nitride to the blocking oxide was higher (compared 
to that from bottom of trapping nitride to tunneling oxide) for the $10 \mathrm{Mrad}$ gamma irradiated MONOS device under NVS. Therefore, the STOB-MONOS device with deeper negative-charge traps in the oxy-nitride at the bottom of the trapping layer, and deeper positive-charge traps in Sirich nitride at the top of the trapping layer, showed better charge-retention reliability characteristics both before and after gamma irradiation than the STD-MONOS devices.

\section{Conclusions}

As shown in the experimental data, adjusting the $\mathrm{Si}-\mathrm{O}-\mathrm{N}$ composition ratio during nitride deposition improved the radiation-induced charging effect and radiation-induced charge retention reliability characteristics of an STOB-MONOS device. The gamma radiation induced a larger decrease in $V_{\mathrm{T}}$ for the STOB-MONOS device than for the standard MONOS device, and the change in $V_{\mathrm{T}}$ for STOB-MONOS after gamma irradiation had a strong correlation to the TID up to $10 \mathrm{Mrad}$ gamma irradiation. The reliability characteristics of $V_{\mathrm{T}}$ retention time before and after gamma irradiation for STOB-MONOS devices were also significantly improved, compared to the standard MONOS device. Therefore, the STOB-MONOS device is thought to have improved charge retention reliability characteristics, both before gamma irradiation and after $10 \mathrm{Mrad}$ gamma irradiation. The performance improvement of radiation-induced charge retention reliability characteristics of STOB-MONOS devices was induced by large, deeper negative-charge traps in the oxy-nitride at the bottom of the trapping layer and large, deeper positive-charge traps in the Sirich nitride at the top of the trapping layer. The results obtained in this study have demonstrated the feasibility of sensing high TID (up to $10 \mathrm{Mrad}$ gamma irradiation) and permanently holding dosimetric information by using STOB-MONOS devices.

\section{Acknowledgements}

The authors thank the National Nano Device Laboratories (NDL), National Tsing Hua University (NTHU), and National Chiao Tung University (NCTU) for providing the instruments for wafer fabrication and testing. This study was funded in part by the National Science Council (NSC).

\section{References}

1 W. C. Hsieh, H. T. Lee, and F. C. Jong: Sensors 14 (2014) 14553.

2 W. C. Hsieh, H. T. Lee, and F. C. Jong: Sensors 4 (2016) 450.

3 A. Gasperin, G. Ghidini, A. Cester, and A. Paccagnella: IEEE Trans. Nucl. Sci. 54 (2007) 1898.

4 B. Draper, R. Dockerty, M. Shaneyfelt, S. Habermehl, and J. Murray: IEEE Trans. Nucl. Sci. 55 (2008) 3202.

5 F. Y. Qiao, X. Yu, and L. Y. Pan: Proc. 19th Int. Symp. Physical and Failure Analysis of Integrated Circuits (IPFA) (IEEE, Singapore, 2012) pp. 1-4.

6 F. Y. Qiao, L. Y. Pan, and J. Xu: IEEE Trans. Nucl. Sci. 61 (2014) 950.

7 S. Bassi and M. Pattanaik: Proc. 18th IEEE Int. Symp. VLSI Design and Test (VDAT) (IEEE, India, 2014) pp. $1-4$.

8 Y. Takahashi, K. Ohnishi, T. Fujimaki, and M. Yoshikawa: IEEE Trans. Nucl. Sci. 46 (1999) 1578.

9 T. R. Oldham and F. B. McLean: IEEE Trans. Nucl. Sci. 50 (2003) 483.

10 Y. Cheng, M. Ding, X. Wu, X. Liu, and K. Wu: 2013 Int. Conf. Solid Dielectrics (IEEE, Italy, 2013) pp. 764767.

11 J. P. Colonna, E. Nowak, and G. Molas: Proc. 27th Int. Conf. Microelectronics (MIEL) (Nis, Serbia, 2010) pp. $1-4$.

12 C. Sandhya, A. B. Oak, N. Chattar, A. S. Joshi, and U. Ganguly: IEEE Trans. Electron. Devices 56 (2009) 3123. 


\section{About the Authors}

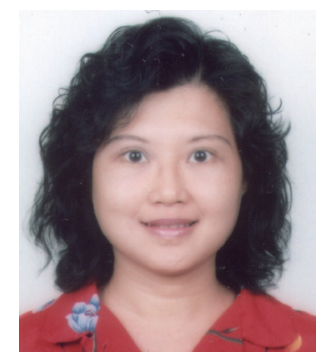

Wen Ching Hsieh received the B.S. degree in Physics from National Cheng Kung University (NCKU), Tainan, Taiwan, in 1986 and the Ph.D. degree in the institute of Nuclear Science from National Tsing Hua University (NTHU), Hsinchu, Taiwan, in 1994. From 1994 to 1995, she was with Winbond Electronics Corporation, Hsinchu, Taiwan, as a research engineer of the memory R\&D division, where she worked on the developement of Flash EEPROM memory devices. In 1996, she joined the memory R\&D division of Taiwan Semiconductor Manufacturing Company, Hsinchu, Taiwan, as a technical manager, where she worked on the simulation and characterization of Flash memory devices. Since 2000, she has been with the department of Opto-Electronic System Engineering in the Minghsin University of Science and Technology (MUST), Hsinchu, Taiwan, where she is currently an assistant professor. She is the supervisor of the semiconductor photo sensor nano device laboratory at MUST. Her research interests are radiation/photo sensor devices using advanced NVM devices processes including high-K gate dielectrics and metal gate. She has published over 50 technical papers.

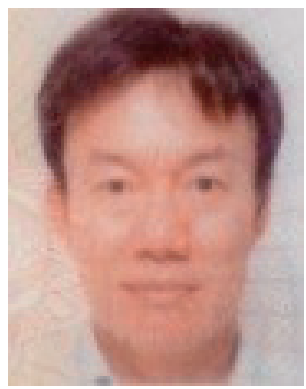

Hao Tien Daniel Lee, IEEE member, has been working mostly in technology startups in the IT industry for more than 20 years, including semiconductor R\&D and production, IC design, and technology innovation. He worked with firms located in UK, US, Germany, and Taiwan. Daniel Lee has acted as a board member, investor, and observer for over 20 venture startups. He was previously involved in the formation of four venture firms, including Vanguard International Semiconductor Corp., Pacific Semiconductor Manufacturing Corp., Pixel Fusion Inc. and ETOMS. Daniel Lee had global operation and engineering development management experience with firms such as

Pixel Fusion Inc. (now as Clear Speed) and Siemens (Memory Division), and had marketing, business, financial development and engineering management experience with ETOMS as well as technology innovation development experience in ERSO/ITRI-Taiwan. Daniel Lee has experience as a consultant in the technology innovative photo-sharing firm UGA Digital Inc. (Huveur Inc.), and RFID solution provider Mutual-Pak Technology Co. Daniel Lee was an adjunct associate professor in Chung Yuan Christian U. and a lecturer in both Submicron-EE of National Chiao Tung University and TCFST of National Tsing Hwa University. As an adjunct associate professor, he has been teaching in the Global Business Program of Business School, Soochow University since 2010. Daniel Lee has also served as an industrial adviser of TAM (Turn Around Management) and BAS (Business Advisory Services) in EBRD (European Bank for Reconstruction and Development) since 2010. Daniel Lee has executive board member experience with firms such as Gabor Vision Corp. and Bison Electronics Inc. Daniel holds a Ph.D. from U. of Connecticut, an M.S. from U. of Utah, and a B.S. from Chung Yuan Christian U. Daniel Lee's specialties include: Technology innovation/incubation and entrepreneur venture start-up management; Operation and technology development management; Technology transfer and international operation; Consumer and memory IC product development; Semiconductor process integration and device research and development. 


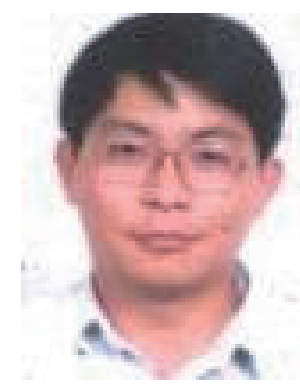

Fuh-Cheng Jong received the B.S. degree in Electrical Engineer from Chinese Culture University (CCU), Taipai, Taiwan, in 1987 and the Ph.D. degree in the institute of Electronics Engineering from National Chiao Tung University (NCTU), Hsinchu, Taiwan, in 1997. From 1994 to 1996, he was with Winbond Electronics Corporation, Hsinchu, Taiwan, as a research engineer of the memory product division, where he worked on the developement of Flash EEPROM memory devices. In 2000, he joined the memory R\&D division of Macronix, Hsinchu, Taiwan, as a technical manager, where he worked on the characterization of Flash memory devices. Since 2002, he has been with the department of Electronic Engineering in the Southern Taiwan University, Tainan, Taiwan, where he is currently an assistant professor.

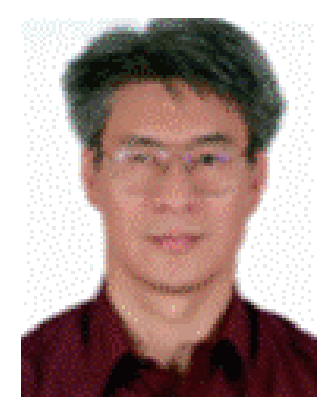

Shich-Chuan Wu received the B.S. degree in Material Science and Engineering National Tsing Hua University, Hsinchu, Taiwan, in 1983 and the M.S. degree in Material Engineering from National Taiwan University, Taipei, Taiwan, in 1986. He received the Ph.D. degree in Electronics Engineering Chiao Tung University, Hsinchu, Taiwan, in 1995. From 1986 to 1990, he was a Research Assistant with the Institute of Material and Electro-Optics, Chung Shan Institute of Science and Technology, Taoyuan County, Taiwan. From 1995 to 1997, he was a post-doctor in the Department of Electrical Engineering National Tsing Hua University, Hsinchu, Taiwan. From 1997 to 2003, he became an associated researcher of National Nano Device Laboratories, Hsinchu, Taiwan, where he is currently a researcher to now. His interest includes the development of semiconductor processing techniques to fabricate nano-scale modules and devices for future applications. 